

\title{
Quasipolynomial Set-Based Symbolic Algorithms for Parity Games
}

\author{
Krishnendu Chatterjee ${ }^{1}$, Wolfgang Dvořák ${ }^{2}$, Monika Henzinger ${ }^{3}$, and Alexander \\ Svozil $^{3 *}$ \\ 1 IST Austria, krish.chat@ist.ac.at \\ 2 Institute of Logic and Computation, TU Wien, dvorak@dbai.tuwien.ac.at \\ 3 Faculty of Computer Science, University of Vienna, \\ \{monika.henzinger, alexander.svozil\}@univie.ac.at
}

\begin{abstract}
Solving parity games, which are equivalent to modal $\mu$-calculus model checking, is a central algorithmic problem in formal methods, with applications in reactive synthesis, program repair, verification of branching-time properties, etc. Besides the standard computation model with the explicit representation of games, another important theoretical model of computation is that of set-based symbolic algorithms. Set-based symbolic algorithms use basic set operations and one-step predecessor operations on the implicit description of games, rather than the explicit representation. The significance of symbolic algorithms is that they provide scalable algorithms for large finite-state systems, as well as for infinite-state systems with finite quotient. Consider parity games on graphs with $n$ vertices and parity conditions with $d$ priorities. While there is a rich literature of explicit algorithms for parity games, the main results for set-based symbolic algorithms are as follows: (a) the basic algorithm that requires $O\left(n^{d}\right)$ symbolic operations and $O(d)$ symbolic space; and (b) an improved algorithm that requires $O\left(n^{d / 3+1}\right)$ symbolic operations and $O(n)$ symbolic space. In this work, our contributions are as follows: (1) We present a black-box set-based symbolic algorithm based on the explicit progress measure algorithm. Two important consequences of our algorithm are as follows: (a) a set-based symbolic algorithm for parity games that requires quasi-polynomially many symbolic operations and $O(n)$ symbolic space; and (b) any future improvement in progress measure based explicit algorithms immediately imply an efficiency improvement in our set-based symbolic algorithm for parity games. (2) We present a set-based symbolic algorithm that requires quasi-polynomially many symbolic operations and $O(d \cdot \log n)$ symbolic space. Moreover, for the important special case of $d \leq \log n$, our algorithm requires only polynomially many symbolic operations and poly-logarithmic symbolic space.
\end{abstract}

*A. S. is fully supported by the Vienna Science and Technology Fund (WWTF) through project ICT15-003. K.C. is supported by the Austrian Science Fund (FWF) NFN Grant No S11407-N23 (RiSE/SHiNE) and an ERC Starting grant (279307: Graph Games). For M.H the research leading to these results has received funding from the European Research Council under the European Union's Seventh Framework Programme (FP/2007-2013) / ERC Grant Agreement no. 340506.

G. Barthe, G. Sutcliffe and M. Veanes (eds.), LPAR-22 (EPiC Series in Computing, vol. 57), pp. 233-253 


\section{Introduction}

In this work, we present new contributions related to algorithms for parity games in the set-based symbolic model of computation.

Parity games. Games on graphs are central in many applications in computer science, especially, in the formal analysis of reactive systems. The vertices of the graph represent states of the system, the edges represent transitions of the system, the infinite paths of the graph represent traces of the system and the players represent the interacting agents. The reactive synthesis problem (Church's problem [18]) is equivalent to constructing a winning strategy in a graph game $[9,46,45]$. Besides reactive synthesis, the game graph problem has been used in many other applications, such as (1) verification of branching-time properties [28], (2) verification of open systems [1], (3) simulation and refinement between reactive systems [43, 31, 2]; (4) compatibility checking [25], (5) program repair [33], (6) synthesis of programs [13]; to name a few. Game graphs with parity winning conditions are particularly important since all $\omega$-regular winning conditions (such as safety, reachability, liveness, fairness) as well as all Linear-time Temporal Logic (LTL) winning conditions can be translated into parity conditions [47, 48]. In a parity game, every vertex of the game graph is assigned a non-negative integer priority from $\{0,1, \ldots, d-1\}$, and a play is winning if the highest priority visited infinitely often is even. Game graphs with parity conditions can model all the applications mentioned above, and are also equivalent to the modal $\mu$-calculus [39] model-checking problem [28]. Thus the parity games problem is a core algorithmic problem in formal methods, and has received wide attention over the decades $[28,5,51,34,58,36,50,12,35]$.

Models of computation: Explicit and symbolic algorithms. For the algorithmic analysis of parity games, two models of computation are relevant. First, the standard model of explicit algorithms, where the algorithms operate on the explicit representation of the game graph. Second, the model of implicit or symbolic algorithms, where the algorithms do not explicitly access the game graph but operate with a set of predefined operations. For parity games, the most relevant class of symbolic algorithms are called set-based symbolic algorithms, where the allowed symbolic operations are: (a) basic set operations such as union, intersection, complement, and inclusion; and (b) one step predecessor (Pre) operations (see [22, 26, 32]).

Significance of set-based symbolic algorithms. We describe the two most significant aspects of set-based symbolic algorithms.

1. Consider large scale finite-state systems, e.g., hardware circuits, or programs with many Boolean variables or bounded-domain integer variables. While the underlying game graph is described implicitly (such as program code), the explicit game graph representation is huge (e.g., exponential in the number of variables). The implicit representation and symbolic algorithms often do not incur the exponential blow-up, that is inevitable for algorithms that require the explicit representation of the game graph. Data-structures such as Binary Decision Diagrams (BDDs) [7] (with well-established tools e.g. CuDD [52]) support symbolic algorithms that are used in verification tools such as NuSMV [19].

2. In several domains of formal analysis of infinite-state systems, such as games of hybrid automata or timed automata, the underlying state space is infinite, but there is a finite quotient. Symbolic algorithms provide a practical and scalable approach for the analysis of such systems: For many applications the winning set is characterized by $\mu$-calculus formulas with one-step predecessor operations which immediately give the desired set-based symbolic algorithms [26, 24]. Thus, the set-based symbolic model of computation is an equally important theoretical model of computation to be studied as the explicit model.

Symbolic resources. In the explicit model of computation, the two important resources are time 
and space. Similarly, in the symbolic model of computation, the two important resources are the number of symbolic operations and the symbolic space.

- Symbolic operations: Since a symbolic algorithm uses a set of predefined operations, instead of time complexity, the first efficiency measure for a symbolic algorithm is the number of symbolic operations required. Note that basic set operations (that only involve variables of the current state) are less resource intensive compared to the predecessor operations (that involve both variables of the current and of the next state). Thus, in our analysis, we will distinguish between the number of basic set operations and the number of predecessor operations.

- Symbolic space: We refer to the number of sets stored by a set-based symbolic algorithm as the symbolic space for the following reason: A set in the symbolic model is considered to be unit space, for example, a set that contains all vertices, or a set that contain all vertices where the first variable is true, represent each $\Theta(n)$ vertices, but can be represented as BDD of constant size. While the size of a set and its symbolic representation is notoriously hard to characterize (e.g., for BDDs it can depend on the variable reordering), in the theoretical model of computation every set is considered of unit symbolic space, and the symbolic space requirement is thus the maximum number of sets required by a symbolic algorithm.

The goal is to find algorithms that minimize the symbolic space (ideally poly-logarithmic) and the symbolic operations.

Previous results. We summarize the main previous results for parity games on graphs with $n$ vertices, $m$ edges, and $d$ priorities. To be concise in the following discussion, we ignore denominators in $d$ in the bounds.

- Explicit algorithms. The classical algorithm for parity games requires $O\left(n^{d-1} \cdot m\right)$ time and linear space $[59,42]$, which was then improved by the small progress measure algorithm that requires $O\left(n^{d / 2} \cdot m\right)$ time and $O(d \cdot n)$ space [34]. Many improvements have been achieved since then, such as the big-step algorithm [50], the sub-exponential time algorithm [36], an improved algorithm for dense graphs [17], and the strategy-improvement algorithm [58], but the most important breakthrough was achieved last year where a quasi-polynomial time $O\left(n^{\lceil\log d\rceil+6}\right)$ algorithm was obtained [12]. While the original algorithm of [12] required quasi-polynomial time and space, a succinct small progress measure based algorithm [35] and value-iteration based approach [29] achieve the quasi-polynomial time bound with quasi-linear space. However, all of the above algorithms are inherently explicit algorithms.

- Set-based symbolic algorithms. The basic set-based symbolic algorithm (based on the direct evaluation of the nested fixed point of the $\mu$-calculus formula) requires $O\left(n^{d}\right)$ symbolic operations and $O(d)$ space [27]. In a breakthrough result [5] presented a set-based symbolic algorithm that requires $O\left(n^{d / 2+1}\right)$ symbolic operations and $O\left(n^{d / 2+1}\right)$ symbolic space (for a simplified exposition see [51]). In recent work [14], a new set-based symbolic algorithm was presented that requires $O\left(n^{d / 3+1}\right)$ symbolic operations and $O(n)$ symbolic space, where the symbolic space requirement is $O(n)$ even with a constant number of priorities.

Open questions. Despite the wealth of results for parity games, many fundamental algorithmic questions are still open. Besides the major and long-standing open question of the existence of a polynomial-time algorithm for parity games, two important open questions in relation to set-based symbolic algorithms are as follows:

- Question 1. Does there exist a set-based symbolic algorithm that requires only quasipolynomially many symbolic operations?

- Question 2. Given the $O(d)$ symbolic space requirement of the basic algorithm, whereas all other algorithms require at least $O(n)$ space (even for a constant number of priorities) 
Table 1: Set-Based Symbolic Algorithms for Parity Games.

\begin{tabular}{lcc}
\hline reference & symbolic operations & symbolic space \\
\hline$[27,59]$ & $O\left(n^{d}\right)$ & $O(d)$ \\
{$[5,51]$} & $O\left(n^{d / 2+1}\right)$ & $O\left(n^{d / 2+1}\right)$ \\
{$[14]$} & $O\left(n^{d / 3+1}\right)$ & $O(n)$ \\
Thm. 2,3 & $n^{O(\log d)}$ & $O(d \log n)$ \\
\hline
\end{tabular}

an important question is: Does there exist a set-based symbolic algorithm that requires $\widetilde{O}(d)$ symbolic space (note that $\widetilde{O}$ hides poly-logarithmic factors), but beats the number of symbolic operations of the basic algorithm? This question is especially relevant since in many applications the number of priorities is small, e.g., in determinization of $\omega$-automata, the number of priorities is logarithmic in the size of the automata [47].

Our contributions. In this work, we not only answer the above open questions (Question 1 and Question 2) in the affirmative but also show that both can be achieved by the same algorithm:

- First, we present a black-box set-based symbolic algorithm based on explicit progress measure algorithm for parity games that use $O(n)$ symbolic space and $O\left(n^{O(\log d)}\right)$ symbolic operations. There are two important consequences of our algorithm: (a) First, given the ordered progress measure algorithm (which is an explicit algorithm), as a consequence of our black-box algorithm, we obtain a set-based symbolic algorithm for parity games that requires quasi-polynomially many symbolic operations and $O(n)$ symbolic space. (b) Second, any future improvement in progress measure based explicit algorithm (such as polynomial-time progress measure algorithm) would immediately imply the same improvement for set-based symbolic algorithms. Thus we answer Question 1 in affirmative and also show that improvements in explicit progress measure algorithms carry over to symbolic algorithms.

- Second, we present a set-based symbolic algorithm that requires quasi-polynomially many symbolic operations and $O(d \cdot \log n)=\widetilde{O}(d)$ symbolic space. Thus we not only answer Question 2 in affirmative, we also match the number of symbolic operations with the current best-known bounds for explicit algorithms. Moreover, for the important case of $d \leq \log n$, our algorithm requires polynomially many symbolic operations and polylogarithmic symbolic space.

We compare our main results with previous set-based symbolic algorithms in Table 1.

Symbolic Implementations. Recently, symbolic algorithms for parity games received attention from a practical perspective: First, three explicit algorithms (Zielonka's recursive algorithm, Priority Promotion [4] and Fixpoint-Iteration [6]) were converted to symbolic implementations [49]. The symbolic solvers had a huge performance gain compared to the corresponding explicit solvers on a number of practical instances. Second, four symbolic algorithms to solve parity games were compared to their explicit versions (Zielonka's recursive algorithm, small progress measure and an automata-based algorithm [40,54]) [55]. For the symbolic versions of the small progress measure, two implementations were considered: (i) Symbolic Small Progress Measure using Algebraic Decision Diagrams [11] and (ii) the Set-Based Symbolic Small Progress Measure [14]. The symbolic algorithms were shown to perform better in several structured instances.

Other related works. Besides the discussed theoretical results on parity games, there are several practical approaches for parity games, such as, (a) accelerated progress measure [23], (b) quasi- 
dominion [4], (c) identifying winning cores [57], (d) BDD-based approaches [37, 38], and (e) an extensive comparison of various solvers [56]. A straightforward symbolic implementation (not set-based) of small progress measure was done in [11] using Algebraic Decision Diagrams (ADDs) and BDDs. Unfortunately, the running time is not comparable with our results as using ADDs breaks the boundaries of the Set-Based Symbolic Model: ADDs can be seen as BDDs which allow the use of a finite domain at the leaves [3]. Recently, a novel approach for solving parity games in quasi-polynomial time which uses the register-index was introduced [41]. Moreover, [41] presents a $\mu$-calculus formula describing the winning regions of the parity game with alternation depth based on the register-index. The existence of such a $\mu$-calculus formula does not immediately imply a quasi-polynomial set-based symbolic algorithm due to constructing the formula using the register-index.

Our work considers the theoretical model of symbolic computation and presents a black-box algorithm as well as a quasi-polynomial algorithm, matching the best-known bounds of explicit algorithms. Thus our work makes a significant contribution towards the theoretical understanding of symbolic computation for parity games.

\section{Preliminaries}

We follow a similar notation as [14].

\subsection{Basic Definitions}

Game Graphs. A game graph is a graph $\Gamma=\left(V, E,\left\langle V_{\mathcal{E}}, V_{\mathcal{O}}\right\rangle\right)$ where the vertices $V$ are partitioned


set of successor vertices of $v$. The set $\operatorname{In}(v)$ describes the set of predecessors of the vertex $v$. More formally $\operatorname{Out}(v)=\{w \in V \mid(v, w) \in E\}$ and $\operatorname{In}(v)=\{w \in V \mid(w, v) \in E\}$. We assume without loss of generality that every vertex has an outgoing edge. We denote the number of vertices with $n$ and the number of edges with $m$.

Plays. Let $\Gamma=\left(V, E,\left\langle V_{\mathcal{E}}, V_{\mathcal{O}}\right\rangle\right)$ be a game graph. Initially, a token is placed on a vertex $v_{0} \in V$. When $v \in V_{z}$ for $z \in\{\mathcal{E}, \mathcal{O}\}$, player $z$ moves the token along one of the edges to a vertex in $\operatorname{Out}(v)$. Formally, a play is an infinite sequence $\left\langle v_{0}, v_{1}, v_{2}, v_{3}, \ldots,\right\rangle$ where for every $i \geq 0$ the following condition holds: $\left(v_{i}, v_{i+1}\right) \in E$.

Parity Game. A parity game $\mathcal{P}$ with $d$ priorities is a game graph $\Gamma$ with a function $\alpha$ that assigns each vertex a priority, i.e., $\alpha: V \mapsto\{0,1,2, \ldots d-1\}$ where $d \in \mathbb{N}$ and $d>0$ and $\mathcal{P}=(\Gamma, \alpha)$. The set $C$ is the set of all priorities. Let $\rho$ be a play of $\mathcal{P}$. Player $\mathcal{E}$ wins $\rho$ if the highest priority occurring infinitely often is even. Player $\mathcal{O}$, on the other hand, wins $\rho$ if the highest priority occurring infinitely often is odd. Let $V_{i}$ for $0 \leq i \leq d-1$ denote the vertices in $\mathcal{P}$ with priority $i$. Formally, we define $V_{i}=\{v \in V \mid \alpha(v)=i\}$.

Strategies. A strategy for player $z \in\{\mathcal{E}, \mathcal{O}\}$ is a function that extends a finite prefix of a play which ends at vertex $v \in V_{z}$ by appending a vertex $v^{\prime} \in \operatorname{Out}(v)$. A memoryless strategy is a strategy that depends only on the last vertex of a play. This corresponds to a function $\sigma_{z}: V_{z} \mapsto V$ such that $\sigma_{z}(v) \in \operatorname{Out}(v)$ for all $v \in V_{z}$. The results from [28, 42] show that it is sufficient to consider memoryless strategies for parity games. We shall therefore from now on only consider memoryless strategies. A starting vertex $s \in V$, a player-E strategy $\sigma$, and a player- $\mathcal{O}$ strategy $\pi$ describe a unique play $\omega(s, \sigma, \pi)=\left\langle v_{0}, v_{1}, v_{2} \ldots\right\rangle$ in a game graph. It is defined as follows: $v_{0}=s$ and for all $i \geq 0$, if $v_{i} \in V_{\mathcal{E}}$ then $v_{i+1}=\sigma\left(v_{i}\right)$ and if $v_{i} \in V_{\mathcal{O}}$ then $v_{i+1}=\pi\left(v_{i}\right)$. 
Quasipolynomial Set-Based Symbolic Algorithms for Parity Games Chatterjee, Dvořák, Henzinger and Svozil

Winning Strategies and Winning Sets. A strategy $\sigma$ is winning at a start vertex $s \in V$ for player $\mathcal{E}$ iff for all strategies $\pi$ of player $\mathcal{O}$, player $\mathcal{E}$ wins the play $\omega(s, \sigma, \pi)$. If there exists a winning strategy at a start vertex $s \in V$ for player $z \in\{\mathcal{O}, \mathcal{E}\}, s$ is part of the winning set of player $z$, $W_{z}$. Every vertex is winning for exactly one of the players [28, 44]. In this work, we study the problem of computing the winning sets for the two players.

\subsection{Symbolic Model of Computation}

In the set-based symbolic model, the game graph is not accessed explicitly but with set-based symbolic operations. The resources in the symbolic model of computation are characterized by the number of set-based symbolic operations and the set-based space.

Set-Based Symbolic Operations. A set-based symbolic algorithm is allowed to use the same mathematical, logical and memory access operations as a regular RAM algorithm, except for the access to the input graph. Given an input game graph $\Gamma=\left(V, E,\left\langle V_{\mathcal{E}}, V_{\mathcal{O}}\right\rangle\right)$ and a set of vertices $S \subseteq V$, the game graph $G$ can be accessed only by the following two types of operations:

1. The basic set operation: $\cup, \cap, \subseteq, \backslash$ and $=$.

2. The one-step operation to obtain the predecessors of the vertices of $S$ in $G$. In particular, we define the predecessor operation

$$
\operatorname{Pre}(S)=\{v \in V \mid \operatorname{Out}(v) \cap S \neq \emptyset\} .
$$

Let $z \in\{\mathcal{E}, \mathcal{O}\}$, then $\bar{z}=\mathcal{O}$ if $z=\mathcal{E}$ and $\bar{z}=\mathcal{E}$ if $z=\mathcal{O}$. The controllable predecessor operation for $z \in\{\mathcal{E}, \mathcal{O}\}$ is defined as

$$
\operatorname{CPre}_{z}(S)=\left\{v \in V_{z} \mid \operatorname{Out}(v) \cap S \neq \emptyset\right\} \cup\left\{v \in V_{\bar{z}} \mid \operatorname{Out}(v) \subseteq S\right\} .
$$

The set $\operatorname{CPre}_{z}(S)$ can be expressed using only Pre and basic set operations. Note that basic set operations (that only involve variables of the current state) are much cheaper compared to the one-step operations (that involve both variables of the current and of the next state). Thus, in our analysis, we will distinguish between the number of basic set operations and the number of one-step operations. Notice that one can define a one-step successor operation (denoted Post $(\cdot)$ ) as well [15], but for the algorithms presented in this work the given predecessor operation suffices.

Set-based Symbolic Space. The basic unit of space for a set-based symbolic algorithm for game graphs are sets [5, 14]. For example, a set can be represented symbolically as one BDD $[7,8,10,21,53,22,20,30,16]$ and each such set is considered as unit space. Consider for example a game graph whose state-space consists of valuations of $N$-Boolean variables. The set of all vertices is simply represented as a true BDD. Similarly, the set of all vertices where the $k$ th bit is false is represented by a BDD which depending on the value of the $k$ th bit chooses true or false. Again, this set can be represented as a constant size BDD. Thus, even large sets can sometimes be represented as constant-size BDDs. In general, the size of the smallest BDD representing a set is notoriously hard to determine and depends on the variable reordering [22]. To obtain a clean theoretical model for the algorithmic analysis, each set is represented as a unit data structure and requires unit space. Thus, for the space requirements of a symbolic algorithm, we count the maximal number of sets the algorithm stores simultaneously and denote it as the symbolic space. 


\subsection{The Progress Measure Algorithm}

High-level intuition. Let $\mathcal{P}=\left(V, E,\left\langle V_{\mathcal{E}}, V_{\mathcal{O}}\right\rangle, \alpha\right)$ be a parity game and let $(\mathcal{W}, \prec)$ be a finite total order with a maximal element $T$ and a minimal element min. A ranking function is a function $f$ which maps every vertex in $V$ to a value in $\mathcal{W}$. The value of a vertex $v$ with respect to the ranking function $f$ is called rank of $v$. The rank $f(v)$ of a vertex $v$ determines how "close" the vertex is to being in $W_{z}$, the winning set of a fixed player $z$. Initially, the rank of every vertex is the minimal value of $\mathcal{W}$. The progress measure algorithm iteratively increases the rank of a vertex $v$ with an operator called Lift with respect to the successors of $v$ and another function called lift. The algorithm terminates when the rank of no vertex can be increased any further, i.e., the least fixed point of Lift is reached. We call the least simultaneous fixed point of all Lift-operators progress measure. When the rank of a vertex is the maximal element of the total order it is declared winning for player $z$. The rest of the vertices are declared winning for the adversarial player $\bar{z}$.

Ranking Function. Let $\mathcal{W}$ be a total order with a minimal element min and maximal element $T$. A ranking function is a function $f: V \mapsto \mathcal{W}$.

The best function. The best function represents the ability of player $z$, given the token is at vertex $v$, to choose the vertex in $O u t(v)$ with the maximal ranking function. Analogously, it constitutes the ability of player $\bar{z}$, given the token is at vertex $v$, to choose the vertex in $\operatorname{Out}(v)$ with the minimal ranking function. Formally, the function best is defined for a vertex $v$ and a ranking function $f$ as follows:

$$
\operatorname{best}(f, v)= \begin{cases}\min \{f(w) \mid w \in \operatorname{Out}(v)\} & \text { if } v \in V_{\bar{z}} \\ \max \{f(w) \mid w \in \operatorname{Out}(v)\} & \text { if } v \in V_{z}\end{cases}
$$

The lift-function. The function lift : $\mathcal{W} \times C \mapsto \mathcal{W}$ defines how the rank of a vertex $v$ is increased according to the rank $r$ of a successor vertex, and the priority $\alpha(v)$ of $v$. The lift function needs to be monotonic in the first argument. Notice that we do not need information about the graph to compute the lift function. In all known progress measures, the lift function is computable in constant time.

The Lift-operation. The Lift-operation potentially increases the rank of a vertex $v$ according to its priority $\alpha(v)$ and the rank of all its successors in the graph. ${ }^{1}$

$$
\operatorname{Lift}(f, v)(u)= \begin{cases}\operatorname{lift}(\operatorname{best}(f, v), \alpha(v)) & \text { if } u=v \\ f(u) & \text { otherwise }\end{cases}
$$

A ranking function is a progress measure if it is the least simultaneous fixed point of all $\operatorname{Lift}(\cdot, v)$-operators.

The Progress Measure Algorithm. The progress measure algorithm initializes the ranking function $f$ with the minimum element of $\mathcal{W}$. Then, the $\operatorname{Lift}(\cdot, v)$-operator is computed in an arbitrary order regarding the vertices. The winning set of player $\mathrm{z}$ can be obtained from a progress measure by selecting those vertices whose rank is $T$. Notice that we need to define the total order $(\mathcal{W}, \prec)$ and a function lift to initialize the algorithm.

For example, the following instantiations of the progress measure algorithm determine the winning set of a parity game: (i) Small Progress Measure [34], (ii) Succinct Progress Measure [35] and the (iii) The Ordered Approach [29]. The running time is dominated by the size of $\mathcal{W}$. For a discussion on the state-of-the-art size of $\mathcal{W}$ we refer the reader to Remark 2.

\footnotetext{
${ }^{1}$ Notice that in the original definition [34] the lift function is applied to all successors and the best of them is chosen subsequently. As the lift is monotone in the first argument the two definitions are equivalent.
} 


\section{Set-Based Symbolic Black Box Progress Measure Algo- rithm}

In this section, we briefly present a basic version of a set-based symbolic progress measure algorithm. The key idea is to compute the Lift-operation with a set-based symbolic algorithm. Then, we improve the basic version to obtain our black box set-based symbolic progress measure algorithm. Finally, we prove its correctness and analyze the symbolic resources.

A basic Black Box Algorithm. Throughout the algorithm, we maintain the family $\mathbf{S}$ of sets of vertices, which contains a set for every element in $\mathcal{W}$, i.e., $\mathbf{S}=\left\{S_{r} \mid r \in \mathcal{W}\right\}$. Intuitively, a vertex $v \in S_{r}$ has rank $f(v)=r$. Initially, we put each vertex into the set with the minimal value of $\mathcal{W}$, i.e., $S_{\min }$. In each iteration, we consider all non-empty sets $S_{r} \in \mathbf{S}$ : The algorithm checks if the ranking function of the predecessors of the vertices in $S_{r}$ must be increased, i.e. $\operatorname{Lift}(f, v)(v) \succ f(v)$ where $v \in \operatorname{Pre}\left(S_{r}\right)$, and if so, performs the Lift-operator for the predecessors. We repeat this step until the algorithm arrives at a fixed point.

Performing a Lift operation. To compute the Lift-operation in the set-based symbolic setting we need to compute two functions for the predecessors of $S_{r}$ : (1) the lift-function and (2) the best-function. By definition, the lift-function does not access the game graph or vertices thereof. Thus we can compute the lift-function without the use of symbolic operations. To compute the best-function we need access to the game graph. In turns out it is simpler to compute the vertices with $\operatorname{best}(f, v) \succeq r$ rather than the vertices with best $(f, v)=r$. Thus, we lift all vertices $v$ with best $(f, v) \succeq r$ to the rank lift $(r, \alpha(v))$. To this end, we first compute the set $S_{\succeq r}=\bigcup_{l \succeq r} S_{l}$ of vertices with rank $\succeq r$. Then, we compute $P=\operatorname{CPre}_{z}\left(S_{\succeq r}\right)$ and, hence, the set $\bar{P}$ comprises the vertices $v \in P$ with $\operatorname{best}(f, v) \succeq r$. Finally, to compute $\operatorname{Lift}(f, v)$, for each $c \in C$, we consider the vertices of $P$ with priority $c$, i.e., the set $\left(P \cap V_{c}\right)$, and add them to the set $S_{\text {lift }(r, c)}$. Notice that we lift each vertex $v$ to lift $(r, \alpha(v))$ where $r=\operatorname{best}(f, v)$ as we consider all non-empty sets $S_{r} \in \mathbf{S}$. No vertex $v$ will be lifted to a set higher than $\operatorname{lift}(r, \alpha(v))$ where $r=b e s t(f, v)$ due to the monotonicity of the lift function in the first argument. If after an iteration of the algorithm a vertex appears in several sets of $\mathbf{S}$ we only keep it in the set corresponding to the largest rank and remove it from all the other sets.

\subsection{Improving the Basic Algorithm}

In this section, we improve the basic Algorithm by (a) reducing the symbolic space from $O(|\mathcal{W}|)$ to $O(n)$ and (b) by reducing the number of symbolic operations required to compute the fixed point.

Key Idea. The naive algorithm considers each non-empty set $S_{r}$ in iteration $i+1$ again no matter if $S_{r}$ has been changed in iteration $i$ or not. Notice that we only need to consider the predecessors of the set $S_{r}$ again when the set $S_{\succeq r}$ in iteration $i+1$ contains additional vertices compared to the set $S_{\succeq r}$ in iteration $i$. To overcome this weakness, we propose Algorithm 1. In this algorithm, we introduce a data structure called $D$. In the data structure $D$ we keep track of the sets $S_{\succeq r}$ instead of the sets $S_{r}$. The set $S_{\succeq r}$ contains all vertices with a rank greater or equal than $r$. Furthermore, we separately keep track of the elements $r \in \mathcal{W}$ where the set $S_{\succeq r}$ changed since the last time $S_{\succeq r}$ was selected to be processed. These elements of $\mathcal{W}$ are called active. Moreover, if we have two sets $S_{\succeq r}=S_{\succeq r^{\prime}}$ with $r \prec r^{\prime}$ there is no need to process the set $S_{\succeq r}$ because lift $\left(r^{\prime}, c\right) \succeq$ lift $(r, c)$ holds due to the monotonicity of the lift function. To summarize, we precisely store a set $S_{\succeq r}$ if there is no $r^{\prime}$ with $r \prec r^{\prime}$ and $S_{\succeq r}=S_{\succeq r^{\prime}}$. Notice, that this instantly gives us a bound on the symbolic space of $O(n)$. 


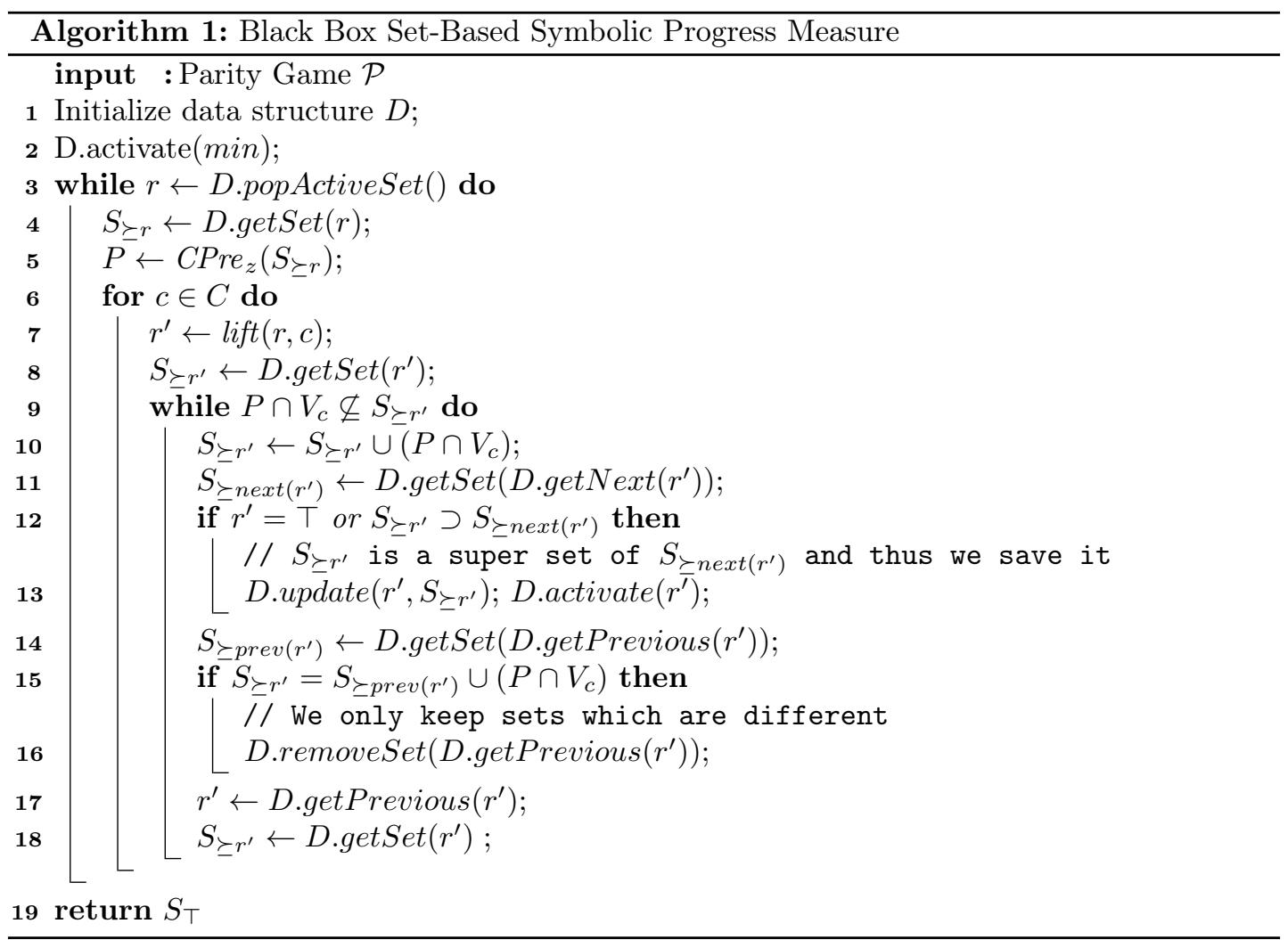

Algorithm Description. In Algorithm 1 we use the data structure $D$ to manage the active $r \in \mathcal{W}$ and in each iteration of the outer while-loop we process the corresponding set $S_{\succeq r}$ of such an $r \in \mathcal{W}$. We first compute $P=\operatorname{CPre}_{z}\left(S_{\succeq r}\right)$, then, for each $c \in C$ we compute $r^{\prime}=\operatorname{lift}(r, c)$ and update the set $S_{\succeq r^{\prime}}$ by adding $P \cap V_{c}$. The inner while-loop ensures that $P \cap V_{c}$ is also added to all the sets $S_{\succeq r^{\prime}}$ with $r^{\prime} \prec r$ and the properties of the data structure are maintained, i.e., (a) all active elements are in the active list, and (b) exactly those $r \in \mathcal{W}$ with $S_{\succeq r} \supset S_{\succeq r^{\prime}}$, for $r \prec r^{\prime}$ are stored in $D$.

Active Elements. Intuitively, an element $r \in \mathcal{W}$ is active if $S_{\succeq r} \supset S_{\succeq r^{\prime}}$, for all $r^{\prime}$ where $r \prec r^{\prime}$ and $S_{\succeq r}$ has been changed since the last time $S_{\succeq r}$ was selected at Line 3 of Algorithm 1. We define active elements more formally later.

Data Structure 1. Our algorithm relies on a data structure D, which supports the following operations:

- D.popActiveSet () returns an element $r \in \mathcal{W}$ marked as active and makes it inactive. If all elements are inactive, returns false.

- D.getSet $(r)$ returns the set $S_{\succeq r}$.

- D.getNext $(r)$ returns the smallest $r^{\prime}$ with $S_{\succeq r} \supset S_{\succeq r^{\prime}}$.

- D.getPrevious $(r)$ returns the largest $r^{\prime}$ where $S_{\succeq r} \subset S_{\succeq r^{\prime}}$.

- D.removeSet $(r)$ marks $r$ as inactive.

- D.activate $(r)$ marks $r$ as active.

- D.update $(r, S)$ updates the set $S_{\succeq r}$ to $S$, i.e., D.getSet $(r)$ returns $S$. Moreover, all sets $S_{\succeq r^{\prime}}$ with $r^{\prime} \prec r$ and $S_{\succeq r^{\prime}}=S_{\succeq r}$ beforehand are updated to $S$ as well. 
Quasipolynomial Set-Based Symbolic Algorithms for Parity Games Chatterjee, Dvořák, Henzinger and Svozil

We initialize D with D.update $(\min , V)$ and D.update $(\top, \emptyset)$.

We can define active elements formally now as the definition depends on $D$.

Definition 1. Let $S_{\succeq r}^{0}=\operatorname{D.getSet}(r)$ be the set stored in $D$ for $S_{\succeq r}$ after the initialization of $D$, and let $S_{\succeq r}^{i}=\bar{D}$.getSet $(r)$ be the set stored in $D$ for $S_{\succeq r}$ after the $i$-th iteration of the while-loop at Line 3. An element $r \in \mathcal{W}$ is active after the $i$-th iteration of the while-loop if $(i)$ for all $r^{\prime} \in \mathcal{W}$ where $r \prec r^{\prime}$ we have $S_{\succ r}^{i} \supset S_{\succ r^{\prime}}^{i}$ and (ii) there is a $j<i$ such that $S_{\succ r}^{i} \supset S_{\succ r}^{j}$ and for all $j<j^{\prime} \leq i$ the set $S_{\succeq r}$ is not selected in Line 3 in the $j^{\prime}$-th iteration. Additionally, we consider $r=\min$ as active before the first iteration. An element $r \in \mathcal{W}$ is inactive if it is not active.

Notice that, in Algorithm 1 an $r \in \mathcal{W}$ is active iff $r$ is marked as active in $D$. The algorithm ensures this in a very direct way. At the beginning only $\min \in W$ is active, which is also marked as active in $D$ in the initial phase of the algorithm. Whenever some vertices are added to a set $S_{\succeq r}$, it is tested whether $S_{\succeq r}$ is larger than its successor and if so $r$ is activated (Lines 12-13). On the other hand, if something is added to the successor of $S_{\succeq r}$ in the data structure $D$ then the algorithm tests whether the two sets are equal and if so $r$ is rendered inactive (Lines 15-16). Implementation of the data structure $D$. The data structure uses an AVL-tree and a doubly linked list called "active list" that keeps track of the active elements. The nodes of the tree contain a pointer to the corresponding set $S_{\succeq r}$ and to the corresponding element in the active list.

- Initialization of the data structure $D$ : Create the AVL tree with the elements min and $\top$. The former points to the set of all vertices and the latter to the empty set. Create the doubly linked list called "active list" as an empty list.

- D.popActiveSet(): Return the first element from the active list and remove it from the active list. If the list is empty, return false.

- D.getSet $(r)$ : Searches the AVL tree for $r$ or for the next greater element (w.r.t. $\succeq)$. Then we return the set by using the pointer we stored at the node.

- D.getNext $(r)$ : First performs D.getSet $(r)$ and then computes the inorder successor in the AVL-tree. This corresponds to the next greater node w.r.t. $\succeq$.

- D.getPrevious $(r)$ : First performs D.getSet $(r)$ and then computes the inorder predecessor in the AVL-tree. This corresponds to the next smaller node w.r.t. $\succeq$.

- D.removeSet $(r)$ : This operation needs the element $r$ to be stored in the AVL tree. Search the AVL tree for $r$. Remove the corresponding element from the active list and the AVL Tree.

- D.activate $(r)$ : This operation needs the element $r$ to be stored in the AVL tree. Add $r$ to the active list and add pointers to the AVL-tree. The element in the active list contains a pointer to the tree element and vice versa.

- D.update $(r, S)$ : Perform $S_{\succeq r} \leftarrow \operatorname{D.getSet}(r)$ : If $r$ is contained in the AVL tree then update $S_{\succeq r}$ to $S$. Otherwise, insert $r$ as a new element and let the element point to $S$.

We initialize the data structure $D$ with $\min \in \mathcal{W}$ and $\top \in \mathcal{W}$. Thus, whenever we query $D$ for a value $r \in \mathcal{W}$ we find it or there exists an $r^{\prime} \succ r$ which is in $D$.

Analysis of the data structure $D$. The data structure can be implemented with an AVL-tree and a doubly linked list called "active list" that keeps track of the active elements such that all of the operations can be performed in $O(\log n)$ : when the algorithm computes D.update $(r, S)$ we store $r$ and a pointer to the set $S$ as a node in the AVL tree. By construction, the algorithm only stores pointers to different sets and when we additionally preserve anti-monotonicity among the sets we only store $\leq n$ sets. Therefore, the AVL tree has only $\leq n$ nodes with pointers 
to the corresponding sets and searching for a set with the operation $\operatorname{D.getSet}(r)$ only adds a factor of $\log n$ to the non-symbolic operations when we store $r$ as key with a pointer to $S_{\succeq r}$ in the AVL-tree. Moreover, we maintain pointers between the elements of the active list and the corresponding vertices in the AVL tree.

Remark 1. The described algorithm is based on a data structure D which keeps track of the sets that will be processed at some point later in time. Note that this data structure does not access the game graph but only stores pointers to sets that the Algorithm 1 maintains. The size of the AVL tree implementing D is proportional to the symbolic space of the algorithm.

\subsubsection{Correctness}

In order to prove the correctness of Algorithm 1 we tacitly assume that the algorithm terminates. An upper bound on the running time is then shown in Proposition 2.

Proposition 1 (Correctness.). Let $\mathcal{P}$ be a parity game. Given a finite total order $(\mathcal{W}, \prec)$ with minimum element min, a maximum element $T$ and a monotonic function lift : $\mathcal{W} \times C \mapsto \mathcal{W}$ Algorithm 1 computes the least simultaneous fixed point of all Lift $(\cdot, v)$-operators.

To prove the correctness of Algorithm 1, we prove that when Algorithm 1 terminates, the function $\rho(v)=\max \left\{r \in \mathcal{W} \mid v \in S_{\succeq r}\right\}$ is equal to the least simultaneous fixed point of all $\operatorname{Lift}(\cdot, v)$-operators. We show that when the properties described in Invariant 1 hold, the function $\rho$ is equal to the least fixed point at the termination of the algorithm. Then, we prove that we maintain the properties of Invariant 1.

Invariant 1. Let $\widetilde{\rho}$ be the least simultaneous fixed point of $\operatorname{Lift}(\cdot, v)$ and $\rho(v)=\max \{r \in \mathcal{W} \mid$ $\left.v \in S_{\succeq r}\right\}$ be the ranking function w.r.t. the sets $S_{\succeq r}$ that are maintained by the algorithm.

1. Before each iteration of the while-loop at Line 3 we have $S_{\succeq r_{2}} \subseteq S_{\succeq r_{1}}$ for all $r_{1} \preceq r_{2}$ (anti-monotonicity).

2. Throughout Algorithm 1 we have $\widetilde{\rho}(v) \succeq \rho(v)$ for all $v \in V$.

3. For all $r \in \mathcal{W}:$ (a) $r$ is active or (b) for all $v \in \operatorname{CPre}_{z}\left(S_{\succeq r}\right)$ : if best $(\rho, v)=r$, then $\rho(v)=\operatorname{Lift}(\rho, v)(v)$.

In the following paragraph, we describe the intuition of Invariant 1. Then, we show that the properties of Invariant 1 are sufficient to obtain the correctness of Algorithm 1. Finally, we prove that each property holds during the while-loop at Line 3.

Intuitive Description. The intuitive description is as follows:

1. Ensures that the sets $S_{\succeq r}$ contain the correct elements. Having the sets $S_{\succeq r}$ allows computing best $(f, v) \succeq r$ as discussed at the beginning of the section.

2. Guarantees that $\rho$ is a lower bound on $\tilde{\rho}$ throughout the algorithm.

3. When an $r \in \mathcal{W}$ is not active, the rank of no vertex can be increased by applying lift to the vertices which have $\operatorname{best}(\rho, v)=r$.

When the algorithm terminates, all $r \in \mathcal{W}$ are inactive and $\rho$ is a fixed point of all $\operatorname{Lift}(\rho, v)$ by condition (3b). The next lemma proves that Algorithm 1 computes the least simultaneous fixed point of all $\operatorname{Lift}(\cdot, v)$ operators for a parity game.

Lemma 1 (The Invariant is sufficient). Let the lift function be monotonic in the first argument and $(\mathcal{W}, \prec)$ be a total order. The ranking function $\rho$ at termination of Algorithm 1 is equal to the least simultaneous fixed point of all Lift $(\cdot, v)$-operators for the given parity game $\mathcal{P}$. 
Proof. Consider the ranking function $\rho(v)=\max \left\{r \in \mathcal{W} \mid v \in S_{\succ r}\right\}$ computed by Algorithm 1 . By Invariant 1(2) we have $\widetilde{\rho}(v) \succeq \rho(v)$ for all $v \in V$. We next show that $\rho(v)$ is a fixed point of $\operatorname{Lift}(\rho, v)$ for all $v \in V$. When the algorithm terminates, no $r \in \mathcal{W}$ is active. Consider an arbitrary $v$ and let $r=$ best $(\rho, v)$. Now, as the set $r$ is not active, by Invariant $1(3 \mathrm{~b})$, we have $\rho(v)=\operatorname{Lift}(\rho, v)(v)$. Thus $\rho(v)$ is a fixed point of $\operatorname{Lift}(\rho, v)$ for all vertices in $V$. Therefore, as $\rho$ is a simultaneous fixed point of all $\operatorname{Lift}(\cdot, v)$-operators and $\widetilde{\rho}$ is the least such fixed point, we obtain $\rho(v) \succeq \widetilde{\rho}(v)$ for all $v \in V$. Hence we have $\rho(v)=\widetilde{\rho}(v)$ for all $v \in V$.

The following lemmas prove each part of the invariant separately. The first part of the invariant describes the anti-monotonicity property which is needed to compute the best function with the $\mathrm{CPre}_{z}$ operator.

Lemma 2. Invariant 1(1) holds: Let $r_{1}, r_{2} \in \mathcal{W}$ and $r_{1} \preceq r_{2}$. Before each iteration of the while-loop at Line 3 we have that if a vertex $v$ is in a set $S_{\succeq r_{2}}$ then it is also in $S_{\succeq r_{1}}$ (anti-monotonicity).

Proof. We prove the claim by induction over the iterations of the while-loop. Initially, the claim is satisfied as the only non-empty set is $S_{\min }$. It remains to show that when the claim is valid at the beginning of an iteration, then the claim also holds in the next iteration. By induction hypothesis, the claim holds for the sets at the beginning of the while-loop. In the trivial case, the algorithm terminates and the claim holds by the induction hypothesis. Otherwise, the sets are only modified at Line 10 and stored at Line 13. First, the vertices $P \cap V_{c}$ are added into the set $S_{\succ r^{\prime}}$. Let $r^{\prime \prime}=D$.getPrevious $\left(r^{\prime}\right)$. Notice that after activating $r^{\prime}$ all $r$ with $r^{\prime \prime} \prec r \prec r^{\prime}$ refer to the same set as $r^{\prime}$ and thus we add $P \cap V_{c}$ implicitly to all $r$. In the next iteration the while-loop then adds $P \cap V_{c}$ also to the set $S_{\succeq r^{\prime \prime}}$. As this done iteratively until a set $S_{\succeq r^{*}}$ with $P \cap V_{c} \subseteq S_{\succeq r^{*}}$ is reached (Lines 9-17), the algorithm ensures that $P \cap V_{c}$ is contained in all set $S_{\succeq r^{\prime \prime}}$ with $r \prec r^{\prime}$. By induction hypothesis we know that the invariant holds for all $r_{2} \succ r^{\prime}$ ( $S_{\succeq r_{2}}$ is unchanged), and as the algorithm added $P \cap V_{c}$ to all set $S_{\succeq r^{\prime \prime}}$ with $r^{\prime \prime} \preceq r^{\prime}$ the claim holds for all $r_{1}, r_{2} \preceq r^{\prime}$.

The second part of the invariant shows that the fixed point Algorithm 1 computes is always smaller or equal to the least fixed point. In particular, the fixed point computed by the algorithm is defined as $\rho(v)=\max \left\{r \in \mathcal{W} \mid v \in S_{\succeq r}\right\}$ and we denote the least fixed point with $\widetilde{\rho}$. The proof is by induction: In the beginning, every vertex is initialized with the minimum element which obviously suffices for the claim. When we apply the lift function to vertices, we observe that by the induction hypothesis the current value of a vertex is below or equal to the fixed point. Additionally, we obtain a rank which is also smaller or equal to the lifted value of $\widetilde{\rho}$ for every vertex as lift is a monotonic function.

Lemma 3. Invariant 1(2) holds: Throughout Algorithm 1 we have $\widetilde{\rho}(v) \geq \rho(v)$ for all $v \in V$.

Proof. Before the while-loop at Line 3 the claim is obviously satisfied as $\widetilde{\rho}(v) \succeq$ min for all $v \in V$. We prove the claim by induction over the iterations of the while-loop: Assume we have $\rho(v) \preceq \widetilde{\rho}(v)$ for all $v \in V$ before an iteration of the while-loop. The function $\rho(\cdot)$ is only changed at Line 10 and stored at Line 13 where the set $\left(P \cap V_{c}\right)$ is added to $S_{\succeq r^{\prime}}$. For $v \in P \cap V_{c}$ we have that $v$ is a priority $c$ vertex and either $v$ is a player- $z$ vertex with a successor in $S_{\succeq r}$ or a player- $\bar{z}$ vertex with all successors in $S_{\succeq r}$. Thus, $r \preceq \operatorname{best}(\rho, v)$ for $v \in P$. At Line 7 we compute the lift-operation for ranking $r$ with priority $c$ which results in the ranking $r^{\prime}$ for the first iteration of the while-loop. By the monotonicity of the lift operation and the induction hypothesis we have that $r^{\prime}=\operatorname{lift}(r, c)(v) \preceq \operatorname{lift}(\operatorname{best}(\rho, v), c)(v) \preceq \operatorname{lift}(\operatorname{best}(\widetilde{\rho}, v), c)=\widetilde{\rho}(v)$ for $v \in P \cap V_{c}$ and thus adding $v$ to $S_{\geq r^{\prime}}$ maintains the invariant (if $\rho(v) \succ r^{\prime}$ beforehand it is not changed and 
otherwise it is lifted to $\left.r^{\prime} \prec \widetilde{\rho}(v)\right)$. In the later iterations of the while-loop $P \cap V_{c}$ is added to sets with smaller $r^{\prime}$, which does not affect $\rho$, as these vertices already appear in sets with larger rank.

The following lemma proves the third part of Invariant 1: Either there is an active $r \in \mathcal{W}$, i.e., the set $S_{\geq r}$ needs to be processed, or $\rho(v)$ is a fixed point. We prove the property again by induction: Initially, the set min $\in \mathcal{W}$ is active and every other set is empty which trivially fulfills the property. Then, in every iteration when we change a set with value $r$ we either activate it, or there is a set with a value $r^{\prime} \succeq r$ where $S_{\succeq r^{\prime}}$ subsumes $S_{\succeq r}$. In the former case, the condition is instantly fulfilled. In the latter case, there is no vertex $v$ where best $(\rho, v)=r$ which renders $S_{\succeq r}$ irrelevant by definition of $\rho$.

Lemma 4. Invariant 1(3) holds: For all $r \in \mathcal{W}$ :

1. $S_{\succeq r}$ is active or,

2. $\forall v \in \operatorname{CPre}_{z}\left(S_{\succeq r}\right)$ : if best $(\rho, v)=r$, then $\rho(v)=\operatorname{Lift}(\rho, v)(v)$

Proof. We prove this invariant by induction over the iterations of the while-loop: Before the while-loop at Line 3 the claim is obviously satisfied as we activate min which contains all vertices; for all other $r \in \mathcal{W}$ the set $S_{\succeq r}$ is empty and thus condition (2) is trivially satisfied.

Assume the condition holds at the beginning of the loop. We can, therefore, assume by the induction hypothesis that the condition holds for all the sets. If there is no active $r \in \mathcal{W}$, the algorithm terminates and the condition holds by the induction hypothesis. The condition for a set $S_{\succeq r}$ can be violated only if either the set $S_{\succeq r}$ is changed or the set $S_{\succeq r}$ is deactivated. That is either at Line 3, Line 10 or Line 16 of the algorithm.

Let us first consider the changes made in the while-loop. If a set $S_{\succeq_{r}}$ is changed in Line 10, then the algorithm either activates $r$ (Line 13) and thus satisfies (1) or $S_{\succeq \text { next }(r)}=S_{\succeq r}$ which implies that best $(\rho, v) \neq r$ and thus (2) is fulfilled trivially. At Line 16 there is no vertex $v$ with best $(\rho, v)=r$ (as there is no vertex $w$ with $\rho(w)=r$ ) and thus (2) is satisfied (and it is safe to remove/deactivate the set in Line 16).

Now consider the case where we remove the set $S_{\succeq r}$ and make $r$ inactive at Line 3 . If the set $S_{\succeq r}$ is unchanged during the iteration of the outer while-loop then $S_{\succeq r}$ satisfies condition (2) after the iteration. This is because for all $v$ with best $(\rho, v)=r$ and $\alpha(v)=c$ we have that if $v$ is not already contained in $S_{\succeq l i f t(r, c)}$ the algorithm adds it to the set $S_{\succeq l i f t(r, c)}$ in Line 10 in the first iteration of the while-loop when processing $c$. This is equivalent to applying $\operatorname{Lift}(\rho, v)(v)=\operatorname{lift}(r, c)$. If the set $S_{\succeq r}$ is changed during the iteration then this happens in the inner while-loop. As argued above, then either $r$ is activated and thus satisfies (1) or $S_{\succeq \text { next }(r)}=S_{\succeq r}$ holds. Thus, there is no vertex $v$ with best $(\rho, v)=r$, i.e., (2) is satisfied.

\subsubsection{Symbolic Resources}

In the following, we discuss the amount of symbolic resources Algorithm 1 needs. We determine the number of symbolic one-step operations, the number of basic set operations and the symbolic space consumption.

Proposition 2. The number of symbolic one-step operations in Algorithm 1 is in $O(n \cdot|\mathcal{W}|)$.

Proof. Each iteration of the while-loop at Line 3 processes an active $r$. That means, that the set $S_{\succeq r}$ was changed in a prior iteration. We use a symbolic one-step operation at Line 5 for each active $S_{\succeq r}$. It, therefore, suffices to count the number of possibly active sets throughout the execution of the algorithm. Initially only $S_{\succeq \min }$ is active. After extracting an active set out of the data structure $D$, it is deactivated at Line 3 . We only activate a set $S_{\succeq x}$ when a new 
vertex is added to it at Line 13 . Because there can only be $n$ vertices with ranking $\succeq x$ for all $x \in \mathcal{W}$ the size of each set $\left|S_{\succeq x}\right|$ is smaller or equal to $n$. In the worst case, we eventually put every vertex into every set $S_{\succeq x}$ where $x \in \mathcal{W}$. Thus we activate $n \cdot|\mathcal{W}|$ sets which is equal to the number of symbolic one-step operations.

A similar argument works for analysing the number of basic set operations.

Proposition 3. The number of basic set operations in Algorithm 1 is in $O(d \cdot n \cdot|\mathcal{W}|)$.

Proof of Proposition 3. As proven in Proposition 2, there are $O(n \cdot|\mathcal{W}|)$ iterations of the outer while-loop and thus $O(n \cdot|\mathcal{W}|)$ iterations of the for-loop. Thus the inner while-loop is started $O(d n \cdot|\mathcal{W}|)$ times. The test whether the while-loop is started only requires two basic set operations and the overall costs are bound by $O(d n \cdot|\mathcal{W}|)$. We bound the overall costs for the iterations of the inner while-loop by an amortized analysis. First, notice that each iteration just requires 8 basic set operations (including testing the while condition afterward). In each iteration for a value $r^{\prime} \in \mathcal{W}$ we charge the $r^{\prime}$ for the involved basic set operations. Notice, that in each such an iteration new vertices are added to the set $S_{\succeq r^{\prime}}$ and thus $r^{\prime}$ is processed at most $n$ times. Thus each $r^{\prime} \in \mathcal{W}$ is charged for at most $8 n$ basic set operations Therefore, the number of basic set operations is $O(d \cdot n \cdot|\mathcal{W}|)+O(n \cdot|\mathcal{W}|)=O(d \cdot n \cdot|\mathcal{W}|)$.

Due to Proposition 1, Proposition 2, Proposition 3 and the fact that we use $\leq n$ sets in the data structure $D$, we obtain Theorem 1 .

Theorem 1. Given a parity game, a finite total order $(\mathcal{W}, \succ)$ and a monotonic function lift we can compute the least fixed point of all Lift $(\cdot, v)$ operators with $O(n \cdot|\mathcal{W}|)$ symbolic one-step operations, $O(d \cdot n \cdot|\mathcal{W}|)$ basic set operations, and $O(n)$ symbolic space.

\section{Implementing the Ordered Progress Measure}

In this section, we plug the ordered approach to progress measure (OPM) described by Fearnley et al. [29] into Algorithm 1. To do this, we recall the witnesses they use in their algorithm and encode it with a specially-tailored technique to obtain an algorithm with a sublinear amount of symbolic space. Finally, we argue that the function lift : $\mathcal{W} \times C \mapsto \mathcal{W}$ and the total order $(\mathcal{W}, \preceq)$ described in [29] can be used to fully implement Algorithm 1 .

The Ordered Progress Measure. To implement the ordered progress measure algorithm we need to argue that the lift-operation is monotonic in the first argument and the order $(\mathcal{W}, \preceq)$ is a total finite order in order to fulfill the conditions of Algorithm 1. Let $\mathcal{P}$ be a parity game and $C=\{0, \ldots, d-1\}$ be the set of priorities in $\mathcal{P}$. The set $\mathcal{W}$ in the ordered progress measure consists of tuples of priorities of length $k$, where $k \in O(\log n)$. Each element in the tuple is an element of $C_{-}=C \cup\left\{\left\{_{-}\right\}\right.$, i.e., it is either a priority or "_". The set $C_{-}$has a total order $\left(C_{-}, \preceq\right)$ such that $\_$is the smallest element, odd priorities are order descending and are considered smaller than even priorities which are ordered ascending. The order $(\mathcal{W}, \preceq)$ is then obtained by extending the order $\left(C_{-}, \preceq\right)$ lexicographically to the tuples $r \in \mathcal{W}$.

For the details of the lift function we refer the reader to the work of Fearnley et al. [29]. An implementation of the lift operation can be found at the GitHub repository of the Oink system [56].

By the results in [29] the order $(\mathcal{W}, \preceq)$ and lift meet the requirements of our algorithms.

Lemma 5. The following holds: (1) The function lift : $\mathcal{W} \times C \mapsto \mathcal{W}$ is monotonic in the first parameter [29, p.6]. (2) The order $(\mathcal{W}, \preceq)$ is a total finite order [29, p.3]. (3) Let $\rho$ be the least 
Quasipolynomial Set-Based Symbolic Algorithms for Parity Games Chatterjee, Dvořák, Henzinger and Svozil

simultaneous fixed point of all Lift $(\cdot, v)$ operators. Then $\rho(v)=\top$ iff player $\mathcal{E}$ has a strategy to win the parity game $\mathcal{P}$ when starting from $v$ [29, Lemma 7.3, Lemma 7.4].

Theorem 1 together with Lemma 5 imply the following theorem.

Theorem 2. Algorithm 1 implemented with the OPM computes the winning set of a parity game with $O(n \cdot|\mathcal{W}|)$ symbolic one-step operations, $O(d \cdot n \cdot|\mathcal{W}|)$ basic set operations, and $O(n)$ symbolic space.

Remark 2. (Bounds for $|\mathcal{W}|$ ). We now discuss the bounds on $|\mathcal{W}|$. The breakthrough result of [12] shows that $|\mathcal{W}|$ is quasi-polynomial $\left(n^{O(\log d)}\right)$ in general and polynomial when $d \leq$ $\log n$. Using the refined analysis of [29], we obtain the following bound on $|\mathcal{W}|$ : in general, $\min \left(n \cdot \log (n)^{d-1}, h \cdot n^{c_{1.45}+\log _{2}(h)}\right)$, where $c_{1.45}=\log _{2}(e)<1.45$ and $h=\lceil 1+d / \log (n)\rceil ;$ and if $d \leq \log n$, then $|\mathcal{W}|$ is polynomial due to [12, Theorem 2.8] and [29, Corollary 8.8]. Note that $O\left(n^{2.45+\log _{2}(d)}\right)$ gives a naive upper bound on $|\mathcal{W}|$ in general. Plugging the bounds in Theorem 2 we obtain a set-based symbolic algorithm that requires quasi-polynomially many symbolic one-step and basic set operations and $O(n)$ symbolic space. The algorithm requires only polynomially many symbolic one-step and basic set operations when $d \leq \log n$.

\section{Reducing the Number of Sets for the OPM}

In this section, we tailor a data structure for the OPM in order to only use $O(d \cdot \log n)$ sets. While each progress measure can be encoded by $\log (|\mathcal{W}|)$ many sets, the challenge is to provide a representation that also allows to efficiently compute the sets $S_{\succeq r}$. Such a representation has been provided for the small progress measure [14] and in the following we adapt their techniques for the OPM.

Key Idea. The key idea of the symbolic space reduction is that we encode the value of each coordinate of the rank $r$ separately. A set no longer just stores the vertices with specific rank $r=b_{1} \ldots b_{k}$ but instead stores all vertices where, say, the first coordinate $b_{1}$ is equal to a specific value in $C_{-}$. This encoding enables us to use only a polylogarithmic amount of symbolic space under the assumption that the number of priorities in the game graph is polylogarithmic in the number of vertices.

Symbolic Space Reduction. Let the rank of $v$ be $r=b_{1} \ldots b_{k}$. Vertex $v$ is in the set $C_{x}^{i}$ iff the $i$ th coordinate of the rank of $v$ is $x$ and a vertex $v$ is in the set $C_{\top}$ iff the rank of $v$ is $T$. Thus $O(\log (n) \cdot d)$ sets suffice to encode all $r \in \mathcal{W}$. We demonstrate this encoding of the sets in Example 1.

Example 1. Let $\mathcal{P}$ be a parity game containing the vertices $v_{1}, v_{2}, v_{3}$. Assume the following ranking function: $f\left(v_{1}\right)=65433, f\left(v_{2}\right)=75422, f\left(v_{3}\right)={ }_{-}-32$. Using the definition of our encoding, we have that: $\left\{v_{3}\right\} \subseteq C_{-}^{1},\left\{v_{1}\right\} \subseteq C_{6}^{1},\left\{v_{2}\right\} \subseteq C_{7}^{1},\left\{v_{3}\right\} \subseteq C^{2},\left\{v_{1}, v_{2}\right\} \subseteq C_{5}^{2},\left\{v_{3}\right\} \subseteq$ $C_{-}^{2},\left\{v_{1}, v_{2}\right\} \subseteq C_{4}^{3},\left\{v_{2}\right\} \subseteq C_{2}^{4},\left\{v_{1}, \bar{v}_{3}\right\} \subseteq C_{3}^{4},\left\{v_{1}\right\} \subseteq C_{3}^{5},\left\{v_{2}, v_{3}\right\} \subseteq C_{2}^{5}$.

Computing the set $S_{\succeq r}$ from $C_{x}^{i}$. We obtain the set $S_{r}$ for rank $r=b_{1} \ldots b_{k}$ with an intersection of the sets $\bigcap_{i=1}^{k} C_{b_{i}}^{i}=S_{r}$. To acquire the set $S_{\succeq r}$ we first consider sets where the first $i$ elements are the equal to $b_{1}, \ldots b_{i}$ but the $i+1$ th element $x$ is $\succ b_{i+1}$.

$$
S_{\succeq r}^{i}=\bigcap_{1 \leq j \leq i} C_{b_{j}}^{j} \cap \bigcup_{x \succ b_{i+1}} C_{x}^{i+1}
$$


Quasipolynomial Set-Based Symbolic Algorithms for Parity Games Chatterjee, Dvořák, Henzinger and Svozil

To construct the set $S_{\succeq r}$ we apply the following union operations:

$$
S_{\succeq r}=\bigcup_{i=1}^{k-1} S_{\succeq r}^{i} \cup S_{r} \cup C_{\top}
$$

That is, we can compute the set $S_{\succeq r}$ with $O(d \cdot \log n)$ set operations and four additional sets. Notice that there is no need to store all sets $S_{\succeq r}^{i}$ as we can immediately add them to the final set when we have computed them. The number of $\cup$-operations is immediately bounded by $O(d \cdot k)=O(d \cdot \log n)$ by the above definitions. In order to bound the number of $\cap$-operations by $O(\log n)$, we do the following. To compute the sets $S_{\succeq r}^{i}$ we introduce an additional set $T^{i}=\bigcap_{1 \leq j \leq i} C_{b_{j}}^{j}$. We have that $S_{\succeq r}^{i}=T^{i} \cap \bigcup_{x \succ b_{i+1}} C_{x}^{i+1}$ and $T^{i+1}=T^{i} \cap C_{b_{i+1}}^{i+1}$, i.e., we just need two $\cap$ operation to compute the next set $S_{\succeq r}^{i+1}$. Moreover, we have that $S_{r}=T^{k}$ and thus can be computed with just one $\cap$ operation. In total, this amounts to $2 k-2=O(\log n)$ many $\cap$-operations.

Updating the set $S_{\succeq r}$ to $S_{\succeq r}^{\prime}$. Assume that the set $S_{\succeq r}$ is the old set that is saved within the sets $C_{x}^{i}$. The new set, $S_{\succeq r}^{\prime}$ is an updated set, which is also a superset. First, compute the difference $S_{\Delta}=S_{\succeq r}^{\prime} \backslash S_{\succeq r}$. Intuitively, the algorithm increased the rank of the vertices in $S_{\Delta}$. We delete their old values by updating $C_{x}^{i}=C_{x}^{i} \backslash S_{\Delta}$ for all $i=0 \ldots k$ and each $x \in\{0, \ldots d-1\}$. Then we add the vertices to the set $C_{r_{i}}^{i}=C_{r_{i}}^{i} \cup S_{\Delta}$ for all $i=0 \ldots k$. In total there are $O(d \log n)$ many \-operations and $O(k)=O(\log n)$ many $\cup$-operations.

Using the above techniques for constructing and updating the sets Algorithm 1 can be modified to obtain the following theorem. We present the details in Section 5.1.

Theorem 3. The winning set of a parity game can be computed in $O(n \cdot|\mathcal{W}|)$ symbolic one-step operations, $O\left(d^{2} n \cdot|\mathcal{W}| \cdot \log n\right)$ basic set operations, and $O(d \cdot \log n)$ symbolic space.

Remark 3. Note that Theorem 3 achieves bounds similar to Theorem 1 with a factor $d \cdot \log n$ increase in basic set operations, however, the symbolic space requirement decreases from $O(n)$ to $O(d \cdot \log n)$. In particular, using the bounds as mentioned in Remark 2, we obtain a setbased symbolic algorithm that requires quasi-polynomially many symbolic one-step and basic set operations, and $O(d \cdot \log n)$ symbolic space, and moreover, when $d \leq \log n$, then the algorithm requires polynomially many symbolic one-step and basic set operations and only poly-logarithmic $O\left(\log ^{2} n\right)$ symbolic space.

Remark 4. Recall that our AVL-tree data structure potentially requires $O(n)$ non-symbolic space (cf. Remark 1) in the worst case. The algorithm above reduces the symbolic space requirement to $O(d \cdot \log n)$. We briefly outline how to reduce the non-symbolic space to the same bound. The main purpose of our AVL-tree data structure is to (a) avoid storing all sets explicitly and (b) efficiently maintain pointers to the active sets. As we now have a succinct representation of all sets we are only left with (b). For (b), we additionally maintain $d \cdot \log n+1$ sets in a data structure $O$ to determine if the set $S_{\succeq r}$ was changed since the last time we chose $r$ at the start of the while-loop which processes an active $r$ in each iteration. In each iteration of the outer while loop the chosen active set $S_{\succ_{r}}$ of $D$ is copied into the corresponding set $T_{\succ r}$ in $O$. We can then identify sets $S_{\succeq r}$ which changed since the last iteration where $r$ was chosen by testing $\left(S_{\succeq r} \neq T_{\succeq r}\right)$. To obtain an active element, it remains to additionally check whether there is no $r^{\prime}>r$ where $S_{\succeq r^{\prime}}=S_{\succeq r}$. If $S_{r} \neq \emptyset$ this property is true and we can return an active set. Because we need to possibly go through $\mathcal{W}$ in each iteration, the number of basic set operations is increased by a factor of $\mathcal{W}$. In total, the number of basic set operations are increased to $O\left(d \cdot n \cdot|\mathcal{W}|^{2} \log n\right)$ while the other symbolic resource consumption stays the same. 


\subsection{Proof of Theorem 3}

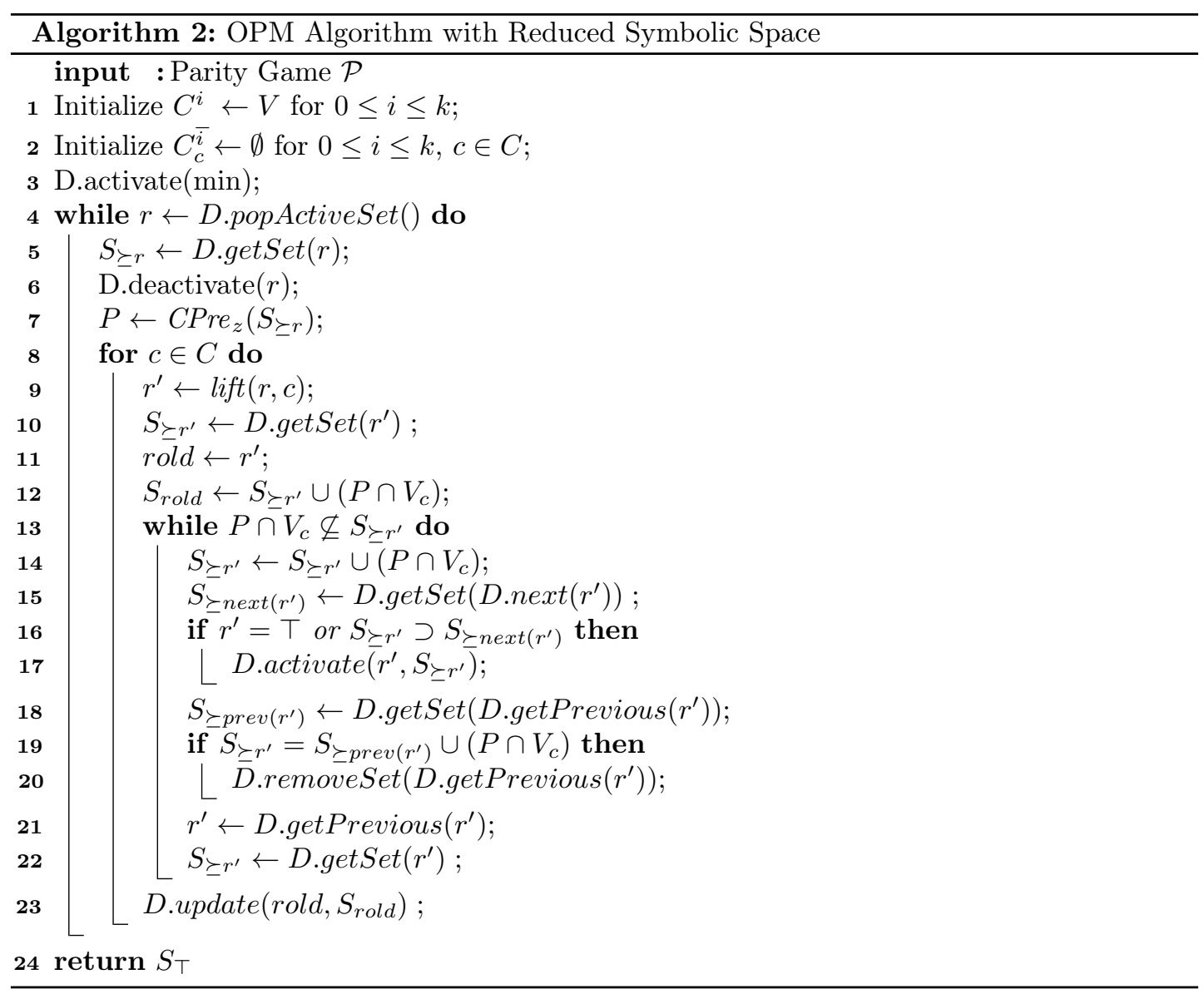

Data Structure. The new data structure $D$ supports all functions of Data Structure 1 but the nodes of the AVL tree no longer store a pointer to a set (but only the value $r$ ). The data structure $D$ stores $d \cdot \log n+1$ sets as described in Section 5, and overrides the D.update $(r, S)$-operation.

- D.update(r,S): Updates the set $S_{\succeq r}$ to be $S$. Every set $S_{\succeq r^{\prime}}$ with $r^{\prime} \prec r$ is updated to $S_{\succeq r^{\prime}} \cup S$.

Implementation of the data structure D.

- D.getSet(r): This function computes the set $S_{\succeq r}$ as described in Section 5 and returns it.

- D.update(r,S): Computes the update of the set $S_{\succeq r}$ with $S$ as described in Section 5 . Precondition: $S_{\succeq r} \subseteq S$.

Notice that Algorithm 2 only differs from Algorithm 1 in (a) the way the D.getSet( $r$ ) method is implemented and (b) how the sets are updated, i.e., the overridden D.update $(r, S)$ method. Hence, to establish the correctness of Algorithm 2, it suffices to show that these two methods do exactly the same as the corresponding operations in Algorithm 1. The correctness then directly follows from Proposition 1. 
Proposition 4 (Correctness). Given a parity game $\mathcal{P}$ Algorithm 2 computes the winning set.

Proof. We show that the D.getSet(r) method (in the interplay with the D.update $(r, S)$ method) in Algorithm 2 returns the same sets as the D.getSet(r) method in Algorithm 1. The correctness then directly follows from Proposition 1.

The proof is by induction. Consider the base case after the initialization of the data structure $D$ and its sets $C_{c}^{i}$. In Algorithm 1 we have that $D \cdot \operatorname{get} S e t(\min )$ would return $V$ and $D \cdot \operatorname{get} \operatorname{Set}(r)$ would return the empty set for min $\prec r$. In Algorithm 2, by the initialization in line 1, we have that $D$.getSet (min) would return $V$ and by the initialization in line 2 we have $\operatorname{D.getSet}(r)$ would return the empty set for $\min \prec r$. Therefore, the base case is satisfied.

Notice that the data structures both in Algorithm 1 and Algorithm 2 are only changed in the for-loop. Assume the claim holds before an iteration of the for-loop. Let $\bar{r}$ be the element of $\mathcal{W}$ currently processed by the outer while-loop, and let $\bar{r}^{\prime}$ the $r^{\prime}$ currently processed by the for-loop. Consider some $r \in \mathcal{W}$. By induction hypothesis, D.getSet $(r)$ coincides in both algorithms beforehand. If $r \succ \bar{r}^{\prime}$ then D.getSet $(r)$ is not affected by the changes in both algorithms and thus D.getSet $(r)$ coincides in both algorithms after the iteration of the for loop. If $r \preceq \bar{r}^{\prime}$ then Algorithm 1 updates the data structure such that $P \cup V_{c}$ is added to the set $S_{\succeq r}$ (the set returned by $D$.getSet $(r)$ ). Now consider Algorithm 2. Here the algorithm adds the set $P \cup V_{c}$ to the set $C_{x}^{i}$ that correspond to $\bar{r}^{\prime}$. As $r \preceq \bar{r}^{\prime}$ there is an $i \geq 0$ such that $r$ and $\bar{r}^{\prime}$ coincide on the first $i$ elements and the set $P \cup V_{c}$ is then contained in the set $S_{\succ r}^{i}$. That means that the set returned by $D$.getSet $(r)$ contains the set $P \cup V_{c}$. Moreover, as only vertices in $P \cup V_{c}$ are affected by the update, all the vertices that were previously contained in $D$.getSet $(r)$ are still contained in the set. In other words, Algorithm 2 adds $P \cup V_{c}$ to the set $S_{\succeq r}$. That is, the two D.getSet $(r)$ methods coincide also after the iteration of the for-loop for all $r \in \mathcal{W}$. Thus, we have that D.getSet $(r)$ coincides in the two algorithms and the correctness of Algorithm 1 extends to Algorithm 2.

Proposition 5 (Symbolic operations). Algorithm 2 uses $O(d \log n)$ sets with $O(n|\mathcal{W}|)$ symbolic one-step operations and $O\left(d^{2} n|\mathcal{W}| \log n\right)$ basic set operations.

Proof. There are $O(n \cdot|\mathcal{W}|)$ iterations if the while-loop at Line 4 (cf. Proposition 2). Therefore, the number of symbolic one-step operations is $O(n \cdot|\mathcal{W}|)$. In each iteration of the while-loop, the for-loop at Line 8 has $d$ iterations. The basic set operations at Line 10, Line 13, Line 12 and at Line 23 occur $O(d \cdot n \cdot|\mathcal{W}|)$ times. This sums up to a total of $O\left(d^{2} \cdot n \cdot|\mathcal{W}| \log n\right)$ basic set operations (as each getSet(r) requires $O(d \log n)$ basic set operations). By the same amortized argument as in the proof of Proposition 2, we obtain that the total number of basic set-operations in executions of the inner while-loop is in $O(n|\mathcal{W}| d \log n)$. The number of basic set operations is, thus, in $O\left(d^{2} n|\mathcal{W}| \log n\right)+O(d n|\mathcal{W}| \log n)=O\left(d^{2} n \cdot|\mathcal{W}| \log n\right)$.

Due to Proposition 4 and Proposition 5 and the fact that we use only $O(d \log n)$ symbolic space in the modified data structure $D$, we obtain Theorem 3 .

\section{Conclusion}

In this work, we present improved set-based symbolic algorithms for parity games. There are several interesting directions for future work. On the practical side, implementations and experiments with case studies, especially for the algorithm presented in Section 3 instantiated with either the ordered approach or the succinct progress measure, is an interesting direction. On the theoretical side, recent work [15] has established lower bounds for symbolic algorithms for graphs, and whether lower bounds can be established for symbolic algorithms for parity games is another interesting direction for future work. 
Quasipolynomial Set-Based Symbolic Algorithms for Parity Games Chatterjee, Dvořák, Henzinger and Svozil

\section{References}

[1] R. Alur, T. A. Henzinger, and O. Kupferman. Alternating-time temporal logic. J. ACM, 49:672-713, 2002.

[2] R. Alur, T.A. Henzinger, O. Kupferman, and M.Y. Vardi. Alternating refinement relations. In CONCUR'98, LNCS 1466, pages 163-178. Springer, 1998.

[3] R. Iris Bahar, Erica A. Frohm, Charles M. Gaona, Gary D. Hachtel, Enrico Macii, Abelardo Pardo, and Fabio Somenzi. Algebraic decision diagrams and their applications. Formal Methods in System Design, 10(2/3):171-206, 1997.

[4] M. Benerecetti, D. Dell'Erba, and F. Mogavero. Solving parity games via priority promotion. In $C A V$, pages 270-290, 2016.

[5] A. Browne, E. M. Clarke, S. Jha, D. E. Long, and W. R. Marrero. An improved algorithm for the evaluation of fixpoint expressions. Theoretical Computer Science, 178(1-2):237-255, 1997.

[6] Florian Bruse, Michael Falk, and Martin Lange. The fixpoint-iteration algorithm for parity games. In GandALF, pages 116-130, 2014.

[7] R. E. Bryant. Graph-based algorithms for boolean function manipulation. IEEE Transactions on Computers, 100(8):677-691, 1986.

[8] R. E. Bryant. Symbolic boolean manipulation with ordered binary-decision diagrams. ACM Comput. Surv., 24(3):293-318, September 1992.

[9] J. R. Büchi and L. H. Landweber. Solving sequential conditions by finite-state strategies. Trans. AMS, 138:295-311, 1969.

[10] J. R. Burch, E. M. Clarke, K. L. McMillan, D. L. Dill, and L. J. Hwang. Symbolic model checking: $10^{\wedge} 20$ states and beyond. In LICS, pages 428-439, 1990.

[11] Doron Bustan, Orna Kupferman, and Moshe Y. Vardi. A measured collapse of the modal $\mu$-calculus alternation hierarchy. In STACS, pages 522-533, 2004.

[12] C. S. Calude, S. Jain, B. Khoussainov, W. Li, and F. Stephan. Deciding parity games in quasipolynomial time. In STOC, pages 252-263, 2017.

[13] P. Cerný, K. Chatterjee, T. A. Henzinger, A. Radhakrishna, and R. Singh. Quantitative synthesis for concurrent programs. In $C A V$, pages 243-259, 2011.

[14] K. Chatterjee, W. Dvořák, M. Henzinger, and V. Loitzenbauer. Improved set-based symbolic algorithms for parity games. In CSL, pages 18:1-18:21, Dagstuhl, Germany, 2017. Schloss DagstuhlLeibniz-Zentrum fuer Informatik.

[15] K. Chatterjee, W. Dvořák, M. Henzinger, and V. Loitzenbauer. Lower bounds for symbolic computation on graphs: Strongly connected components, liveness, safety, and diameter. In SODA, pages 2341-2356. SIAM, 2018.

[16] K. Chatterjee, M. Henzinger, M. Joglekar, and N. Shah. Symbolic algorithms for qualitative analysis of Markov decision processes with büchi objectives. Form. Methods Syst. Des., 42(3):301-327, 2013. Announced at CAV'11.

[17] K. Chatterjee, M. Henzinger, and V. Loitzenbauer. Improved Algorithms for One-Pair and $k$-Pair Streett Objectives. In LICS, pages 269-280, 2015.

[18] A. Church. Logic, arithmetic, and automata. In ICM, pages 23-35, 1962.

[19] A. Cimatti, E. Clarke, F. Giunchiglia, and M. Roveri. Nusmv: a new symbolic model checker. International Journal on Software Tools for Technology Transfer, 2(4):410-425, Mar 2000.

[20] E. Clarke, O. Grumberg, S. Jha, Y. Lu, and H. Veith. Counterexample-guided abstraction refinement for symbolic model checking. J. ACM, 50(5):752-794, September 2003. Announced at CAV'00.

[21] E. M. Clarke, K. L. McMillan, S. V. Aguiar Campos, and V. Hartonas-Garmhausen. Symbolic model checking. In $C A V$, pages 419-427, 1996.

[22] E.M. Clarke, O. Grumberg, and D. Peled. Symbolic model checking. In Model Checking. MIT Press, 1999. 
Quasipolynomial Set-Based Symbolic Algorithms for Parity Games Chatterjee, Dvořák, Henzinger and Svozil

[23] L. de Alfaro and M. Faella. An accelerated algorithm for 3-color parity games with an application to timed games. In $C A V$, pages 108-120, 2007.

[24] L. de Alfaro, M. Faella, T. A. Henzinger, R. Majumdar, and M. Stoelinga. The element of surprise in timed games. In CONCUR, pages 142-156, 2003.

[25] L. de Alfaro and T. A. Henzinger. Interface theories for component-based design. In EMSOFT, pages 148-165. Springer, 2001.

[26] L. de Alfaro, T. A. Henzinger, and R. Majumdar. Symbolic algorithms for infinite-state games. In CONCUR, pages 536-550, 2001.

[27] E. A. Emerson and Ch.-L. Lei. Efficient model checking in fragments of the propositional mu-calculus. In $L I C S$, pages 267-278, 1986.

[28] E.A. Emerson and C.S. Jutla. Tree automata, mu-calculus and determinacy. In FOCS, pages 368-377, 1991.

[29] J. Fearnley, S. Jain, S. Schewe, F. Stephan, and D. Wojtczak. An ordered approach to solving parity games in quasi polynomial time and quasi linear space. In Hakan Erdogmus and Klaus Havelund, editors, SPIN, Santa Barbara, CA, USA, July 10-14, 2017, pages 112-121. ACM, 2017.

[30] R. Gentilini, C. Piazza, and A. Policriti. Symbolic graphs: Linear solutions to connectivity related problems. Algorithmica, 50(1):120-158, 2008. Announced at SODA'03.

[31] T. A. Henzinger, O. Kupferman, and S. K. Rajamani. Fair simulation. Information and Computation, 173(1):64-81, 2002.

[32] T. A. Henzinger, R. Majumdar, and J.F. Raskin. A classification of symbolic transition systems. ACM Trans. Comput. Log., 6(1):1-32, 2005.

[33] B. Jobstmann, A. Griesmayer, and R. Bloem. Program repair as a game. In $C A V$, pages 226-238, 2005.

[34] M. Jurdziński. Small Progress Measures for Solving Parity Games. In STACS, pages 290-301, 2000.

[35] M. Jurdziński and R. Lazic. Succinct progress measures for solving parity games. In LICS, pages $1-9,2017$.

[36] M. Jurdziński, M. Paterson, and U. Zwick. A Deterministic Subexponential Algorithm for Solving Parity Games. SIAM J. Comput., 38(4):1519-1532, 2008.

[37] G. Kant and J. van de Pol. Efficient instantiation of parameterised boolean equation systems to parity games. In GRAPHITE 2012, pages 50-65, 2012.

[38] G. Kant and J. van de Pol. Generating and solving symbolic parity games. In GRAPHITE 2014, pages $2-14,2014$.

[39] D. Kozen. Results on the propositional $\mu$-calculus. Theoretical Computer Science, 27(3):333-354, 1983.

[40] Orna Kupferman and Moshe Y. Vardi. Weak alternating automata and tree automata emptiness. In $S T O C$, pages $224-233,1998$.

[41] Karoliina Lehtinen. A modal $\mu$ perspective on solving parity games in quasi-polynomial time. In LICS, pages 639-648, 2018.

[42] R. McNaughton. Infinite games played on finite graphs. Annals of Pure and Applied Logic, 65(2):149-184, 1993.

[43] R. Milner. An algebraic definition of simulation between programs. In Second International Joint Conference on Artificial Intelligence, pages 481-489. The British Computer Society, 1971.

[44] A. W. Mostowski. Games with forbidden positions. Technical Report 78, University of Gdańsk, 1991.

[45] A. Pnueli and R. Rosner. On the synthesis of a reactive module. In POPL, pages 179-190. ACM Press, 1989.

[46] P.J. Ramadge and W. Murray Wonham. Supervisory control of a class of discrete-event processes. 
SIAM J. Control Optim., 25(1):206-230, 1987.

[47] S. Safra. On the complexity of $\omega$-automata. In FOCS, pages 319-327, 1988.

[48] S. Safra. Complexity of automata on infinite objects. PhD thesis, Weizmann Institute of Science, 1989.

[49] L. Sanchez, J.W. Wesselink, and T.A.C. Willemse. BDD-based parity game solving: a comparison of Zielonka's recursive algorithm, priority promotion and fixpoint iteration. Computer science reports. Technische Universiteit Eindhoven, 2018.

[50] S. Schewe. Solving parity games in big steps. In FSTTCS, pages 449-460. Springer, 2007.

[51] H. Seidl. Fast and simple nested fixpoints. Information Processing Letters, 59(6):303-308, 1996.

[52] F. Somenzi. Colorado university decision diagram package. http://vlsi.colorado.edu/pub/, 1998.

[53] F. Somenzi. Binary Decision Diagrams. In Calculational System Design, NATO Science Series F: Computer and Systems Sciences, volume 173, pages 303-366. IOS Press, 1999.

[54] Antonio Di Stasio, Aniello Murano, Giuseppe Perelli, and Moshe Y. Vardi. Solving parity games using an automata-based algorithm. In CIAA, pages 64-76, 2016.

[55] Antonio Di Stasio, Aniello Murano, and Moshe Y. Vardi. Solving parity games: Explicit vs symbolic. In $C I A A$, pages $159-172,2018$.

[56] T. van Dijk. Oink: An implementation and evaluation of modern parity game solvers. In TACAS, pages 291-308, 2018.

[57] S. Vester. Winning cores in parity games. In LICS, pages 662-671, 2016.

[58] J. Vöge and M. Jurdziński. A discrete strategy improvement algorithm for solving parity games. In $C A V$, pages $202-215,2000$.

[59] W. Zielonka. Infinite games on finitely coloured graphs with applications to automata on infinite trees. Theoretical Computer Science, 200(1-2):135-183, 1998. 